\title{
Myocardial Microvascular Physiology in Acute and Chronic Coronary Syndromes, Aortic Stenosis, and Heart Failure
}

\author{
Alf I. Larsen $\left(\mathbb{D},{ }^{1}\right.$ William F. Fearon, ${ }^{2}$ Todd J. Anderson $\mathbb{D}^{,}{ }^{3}$ and Nico Pijls ${ }^{4}$ \\ ${ }^{1}$ Department of Cardiology, Stavanger University Hospital, University of Bergen, Stavanger, Norway \\ ${ }^{2}$ Stanford University, Stanford, CA, USA \\ ${ }^{3}$ Libin Cardiovascular Institute, Cumming School of Medicine, University of Calgary, Calgary, Canada \\ ${ }^{4}$ Catharina Hospital, Eindhoven, Netherlands \\ Correspondence should be addressed to Alf I. Larsen; alf.inge.larsen@sus.no
}

Received 28 July 2021; Accepted 28 July 2021; Published 26 January 2022

Copyright ( $\odot 2022$ Alf I. Larsen et al. This is an open access article distributed under the Creative Commons Attribution License, which permits unrestricted use, distribution, and reproduction in any medium, provided the original work is properly cited.

Myocardial ischemia occurs when myocardial oxygen demand exceeds the coronary blood supply. The etiology is usually atherosclerotic obstructive epicardial coronary artery disease (CAD) presenting with the features of chronic coronary syndrome (CCS). Fractional flow reserve (FFR) has become the gold standard for assessing myocardial ischemia due to coronary artery stenosis. As demonstrated in the DEFER study, long-term prognosis after deferral of PCI of an intermediate coronary stenosis based on FFR $>/=0.75$ is excellent. The risk of cardiac death or myocardial infarction related to this stenosis is $<1 \%$ per year and not decreased by stenting [1]. In the FAME study, it was shown that routine guidance of revascularization with measurement of FFR in patients with multivessel coronary artery disease, who are undergoing PCI with drug-eluting stents, significantly reduced the rate of the composite endpoint of death, nonfatal myocardial infarction, and repeat revascularization at 1 year [2]. In the FAME 2 trial, the investigators extended their findings by showing that in patients with stable coronary artery disease, FFR-guided PCI, as compared with medical therapy alone, improved the outcome. Furthermore, patients without ischemia had a favorable outcome with medical therapy alone [3].

These studies are dependent on the use of full hyperemia using vasodilating agents like adenosine. As a non-hyperemic surrogate to FFR, the so-called instantaneous wave-free ratio (iFR) and a number of comparable non-hyperemic pressure indices have been proposed [4]. Although these indices have been demonstrated to be noninferior to FFR in studies in relatively low-risk patients (Define Flair [5] and Swede Heart [6]), these are not as well validated and lack the clinical outcome data existing for FFR [7]. In the current issue of the journal, Ebihara et al. explored the effect of rate pressure product (RPP) on instantaneous wave-free ratio (iFR) [8]. By adding these extra parameters, the values might be more accurate and reproducible. They found that the best cutoff value of the iFR for predicting an FFR of 0.8 was 0.90 for all lesions. However, when the study population was divided into the low-RPP and high-RPP groups according to the median RPP, they found different iFR values predicting an FFR of $0.8,0.93$ for the low-RPP group and 0.82 for the high-RPP group. Consequently, the RPP has been demonstrated to affect the relationship between the FFR and iFR. With FFR as the gold standard, the iFR may underestimate and overestimate the functionality of ischemia in the lowand high-RPP groups, respectively.

However, with this being said, the angiographic evidence of "normal" or mildly diseased epicardial coronary arteries, usually defined as the absence of a luminal diameter reduction of $<50 \%$ (or $<70 \%$ of the luminal area reduction), is a common finding. This condition is usually defined as ischemia with no obstructive coronary artery (INOCA) disease and is likely related to the so-called coronary microcirculatory or microvascular dysfunction (CMD). Angina with no obstructive coronary arteries (ANOCAs) is the clinical term when a clinical diagnosis of ischemia is made in a patient without significant obstructive coronary artery disease, without the necessity of having demonstrated 
inducible ischemia. In reported studies on ANOCA, ischemia has been demonstrated in approximately $50 \%$ of the patients. Nevertheless, the two terms INOCA and ANOCA are often interchangeably used.

\section{Epidemiology}

Up to $40 \%$ of patients undergoing coronary angiography with signs and symptoms of angina pectoris are characterized with INOCA [9]. In the American College of Cardiology National Cardiovascular Data Registry from January 2004 through April 2008, at 663 hospitals, slightly more than one-third of patients without known disease who underwent elective cardiac catheterization had obstructive coronary artery disease. The authors of this report suggested better strategies for risk stratification to inform decisions and to increase the diagnostic yield of cardiac catheterization in routine clinical practice [7]. Moreover, estimates from the WISE database indicate that there are at least 3-4 million patients in the USA with signs and symptoms of ischemia despite no evidence of obstructive CAD [10]. However, this might be an old fashion approach to the problem of recurrent angina. In patients with residual angina or recurrence of angina after percutaneous coronary intervention (PCI), functional mechanisms are responsible for the vast majority of cases [11]. The large proportion of patients with angina and near-normal or normal coronary angiogram is thus a large challenge for the cardiology society because a vast number of patients are not appropriately diagnosed. Recently, a large study using intracoronary flow wires in 151 patients with INOCA demonstrated microvascular dysfunction in approximately $75 \%$ of the patients [12].

\section{Definition}

In 1988, Cannon and Epstein proposed that dysfunction of small intramural prearteriolar coronary arteries might be the pathogenic cause of a syndrome introduced as "microvascular angina" (MVA) in this patient population. This condition was characterized by heightened sensitivity of the coronary microcirculation to vasoconstrictor stimuli and a limited microvascular vasodilator capacity [13], mainly caused by dysfunction of small intramural prearteriolar coronary arteries [14]. Tests to identify this syndrome are typically performed using mediators of full hyperemia-adenosine or dipyridamole. However, functional etiology for angina also comprises endothelial dysfunctionassociated vasospasm of large epicardial arteries. In 1959, Prinzmetal and his colleagues described a syndrome characterized by angina at rest, with transient ST-segment elevation, in patients with diseased coronary arteries [15].

This might be diagnosed during provocation tests with acetylcholine during coronary angiography. Acetylcholine binds vascular muscarinic acetylcholine receptors inducing endothelial NO release with subsequent arterial dilatation when endothelial function is intact. However, in the presence of endothelial dysfunction, acetylcholine induces conduit vessel arterial constriction due to direct smooth muscle cell constriction.
Reproduction of typical symptoms, ECG changes, and angiographically verified vasospasm is diagnostic [16]. Unlike the focal spasm and ST elevation seen with classic Prinzmetal's angina, diffuse vasospasm is the usual pattern with endothelial dysfunction detected with acetylcholine. In the CorMica study, a vasospastic pattern was seen in about $1 / 4$ of the INOCA cohort [17]. Many subjects will have both microvascular and conduit vessel abnormalities. Finally, typical symptoms and ECG alterations might also occur without obvious changes of the coronary angiogram in response to acetylcholine indicating small-vessel vasospasm. All of these conditions are more or less associated with the progressive process of coronary atherosclerosis initiated by endothelial dysfunction. It is also important for clinicians to be aware that the vast majority of patients presenting with chest pain and minimal CAD do have an underlying abnormality of coronary vasomotion even if they do not have manifest ischemia on noninvasive testing. This is still not widely recognized amongst the cardiology community. Interventional cardiologists doing diagnostic procedures are critical in conveying this message to patients and referring physicians.

In addition, in a substantial proportion of patients with acute coronary syndromes, normal or near-normal coronary angiograms are found [18]. This condition is known as myocardial infarction with no obstructive coronary arteries (MINOCAs). Moreover, microvascular dysfunction is a major player in the no-reflow phenomenon in primary percutaneous coronary intervention (PCI) for STEMI [19]. However, in this issue, we focus on the microvascular dysfunction described above.

\section{Etiology}

Risk factors for MVD are the same as for CCS. Endothelial dysfunction is the first step in this process, and inflammation is central in the progression of the disease. Patients with coronary endothelial dysfunction are recognized to have significant health service use and morbidity as well as an increased risk of developing flow-limiting coronary artery disease and myocardial events, including death [20].

Additionally, recent studies have shown that especially vasospastic angina is associated with an early inflammatory coronary artery condition documented with the presence of low-grade inflammation-related endothelial dysfunction with resulting diffuse intimal thickening and impaired nitric oxide production [21]. Endothelial dysfunction, the precursor for CAD, is associated with MVD [22]. However, also nonendothelial-dependent vascular dysfunction is associated with the typical risk factors for atherosclerosis like aging [23], hypertension [24], diabetes [25], dyslipidemia, and insulin resistance [26]. The mechanisms underlying the development of MVD are thus multifactorial and only partly explained by current research.

In a small mechanistic study following PCI, both largeand small-vessel vasoconstriction were seen as manifested by a reduction in coronary conduit vessel diameter and in $\mathrm{CBF}$. These effects were reversed by NTG. Serum levels of LDL were modestly related to the reduction of $\mathrm{CBF}$ and to the 
degree of NTG-induced vasodilatation of the coronary microvasculature [27].

\section{Classification}

In 2007, Camici and Crea presented a clinical classification with 4 subtypes of coronary microvascular dysfunction on the basis of the clinical settings in which it occurs: dysfunction occurring in the absence of CAD and myocardial diseases, dysfunction in the presence of myocardial diseases, dysfunction in the presence of obstructive epicardial CAD, and iatrogenic dysfunction [28].

The paper by Zelis et al. in the current issue sheds light on the coronary microvascular dysfunction in the presence of myocardial disease, i.e., aortic stenosis (AS) [29]. They describe the disadvantages of secondary cardiomyopathy in AS: diastolic dysfunction, insufficient capillary density, and diffuse fibrosis.

They refer to the area under the aortic (or, in situations of aortic stenosis, LV) curve during systole (systolic pressure time integral (SPTI)), which has been shown in animal models to have a very high and direct correlation with myocardial oxygen demand, even superior to the rate pressure product [30]. Furthermore, they refer to the diastolic pressure time integral (DPTI) which is an analog for "coronary perfusion pressure." The ratio of these DPTI/SPTI balances supply and demand into a single unitless ratio, although this formulation ignores other factors such as arterial oxygen content and relative LV mass and wall tension [31].

After reviewing the available literature, they found that existing data support an increase in hyperemic flow after TAVI due to a change in the myocardial load line. This change is due to a reduction in wedge pressure, largely "reflecting" LV filling pressures that fall after AS has been treated.

\section{Diagnosis and Methods}

Established diagnostic tools for assessing microvascular disease are not readily available in most cath. labs, leaving many patients with no or a wrong diagnosis. Therefore, a growing part of the interventional cardiology community is looking for an available means to diagnose and quantify microvascular dysfunction to find the appropriate and accurate diagnosis for the individual patient.

For the last 2 decades, studies employing positron emission tomography (PET) have been used to describe the normal range of absolute myocardial blood flow (MBF, $\mathrm{mL}$ / $\mathrm{min} / \mathrm{g}$ ) and of coronary flow reserve (CFR). This is a measure of coronary circulatory capacity defined as the ratio of $\mathrm{MBF}$ during maximal coronary vasodilatation to baseline $\mathrm{MBF}$ [32].

The invasive methods presently used to assess microvascular function, CFR and Index of Microvascular Resistance (IMR), are operator dependent and are based on adenosine to induce hyperemia. In the current issue, Keullards et al. reviewed the new thermodilution-based method for the measurement of absolute coronary blood flow and microvascular resistance [33]. The measurements are easy to perform using the Rayflow ${ }^{\circledR}$ infusion catheter and Coroventis ${ }^{\circledR}$ software. The method is accurate, reproducible, and completely operator independent and has been validated noninvasively against the current golden standard for flow assessment: PET-CT [34].

It has recently been shown that a comprehensive invasive assessment of these patients at the time of coronary angiography can be performed safely and provides important diagnostic information that may affect treatment and outcomes [35]. This should be integrated in modern invasive diagnostics in that conventional stress testing is insufficient for identifying occult coronary abnormalities that are frequently present in patients with angina in the absence of obstructive CAD. A normal noninvasive test for ischemia does not rule out a nonobstructive coronary etiology of angina, nor does it negate the need for comprehensive invasive testing [36].

In addition to PET, intracoronary Doppler measurements are considered close to the gold standard for determining CFR. However, both types of examinations are associated with a certain load of ionizing radiation in addition to the obvious invasive nature of intracoronary Doppler measurements. In the search for less-invasive methods, transthoracic Doppler echocardiography (TTDE) has emerged as a robust method to assess CRF [37].

In the current issue of the journal, Bechsgaard and Prescott described the method of TTDE for assessing coronary flow velocity reserve [38] as an established method of assessment of coronary microvascular function with a well-documented prognostic significance [39]. They review the use of adenosine infusion as a microvascular dilatator by activation of A2A receptors yielding a 3- to 4fold increase in coronary blood flow in a normal epicardial vessel [40]. Furthermore, they describe the ratio of hyperemic to resting coronary flow velocity, coronary flow velocity reserve (CFVR), as an established physiological estimate of coronary microvascular function, which is closely correlated with CFVR measured using an intracoronary Doppler guidewire in patients undergoing angiography for suspected obstructive CAD [41]. Dobutamine cMRI stress can yield useful information about wall motion abnormalities, and ischemia can be assessed using adenosine in INOCA subjects [42]. Due to the lack of radiation of both TTDE and cMRI, these methods might be particularly advantageous for young women with chest pain syndrome requiring diagnostic work-up.

\section{Prognosis}

Patients with MVD show persistence and even worsening of symptoms over time [43], and they constitute a therapeutic problem with considerable residual morbidity associated with functional limitations and reduced quality of life in addition to the increasing economic burden of the health authority system [44]. Impaired CFR is associated with increased mortality in patients with INOCA $[45,46]$. 
Furthermore, impaired CFR without any concomitant impairment of regional or global left ventricular function has additional prognostic significance [47]. In a large study evaluating the prognostic significance of both stress myocardial blood flow $(\mathrm{MBF})$ and myocardial flow reserve (MFR) and the ratio of stress to rest MBF [48], the researchers found MFR to be substantially more consistent, regardless of the choice of input function derivation method and the extraction model used [49].

The link between MVD and flow-mediated vasodilation is further underlined in regard to prognosis in a study evaluating hyperemic velocity, the stimulus for flow-mediated dilation. Hyperemic velocity was a significant risk marker for adverse cardiovascular outcomes. The prognostic value is additive to traditional risk factors and carotid intima-media thickness [50]. This suggests that microvascular dysfunction may be systemic, and that peripheral testing may be useful in diagnosis and prognosis.

The size of the problem and the lack of therapeutic intervention justify the increasing efforts to develop diagnostic tools and to identify new treatment strategies when assessing patients with INOCA.

\section{Treatment}

Reduced physical activity is one of the major avoidance behaviours in patients with coronary heart disease [51]. On the other hand, several studies have documented the positive effect of exercise training (ET) in this population [52]. Psychological morbidity with great impact on daily living is well known in both patients with cardiovascular disease and in patients with chest pain with no obvious physical disease. This includes patients with INOCA. These patients constitute a relatively large proportion of patients taken care of by the health authority system, indicating that this issue has economic consequences for the society that is not neglectable [2].

Therefore, a major end point in the treatment of these patients is symptom control [53].

In patients with MVA, lifestyle modifications such as smoking cessation and weight loss, which are known to improve endothelial dysfunction, are as essential as in the prevention and treatment of CAD [54]. Notably, exercise training has been shown to improve symptoms in this population [55]. A small observational study also indicates that an improvement in $\mathrm{VO}_{2}$ peak is associated with increased CFR and improved endothelial function. Importantly [56], these effects were followed by an improvement in quality of life [57].

Long-term treatment with carvedilol can significantly increase coronary flow reserve and reduce the occurrence of stress-induced perfusion defects, suggesting a favorable effect of the drug on coronary microvascular function in patients with IDC [58]. Additionally, Neglia and coworkers showed a beneficial effect of perindopril on coronary blood flow after 6 months of perindopril treatment. This treatment has also been shown to improve myocardial blood flow and reverse remodeling in myocardial arterioles in spontaneous hypertensive rats [59]. A large multicentre, prospective, randomized, blinded outcome study evaluating intensive medical therapy including high-intensity statins, ACE-Is or
ARBs, and aspirin, vs. usual care in 4422 symptomatic women with INOCA, (Women's IschemiA TRial to Reduce Events In Non-ObstRuctive CAD, (WARRIOR)), will probably give the answer if patients with MVD should be treated as patients with CAD [60].

The purpose of the CorMica trial was to evaluate whether an interventional diagnostic procedure (IDP) linked to stratified medicine improves health status in patients with INOCA. Patients without angiographical obstructive CAD ( $n=151-39 \%)$ were immediately randomized $1: 1$ to the intervention group (stratified medical therapy) or the control group (standard care, IDP sham procedure). The IDP consisted of guidewirebased assessment of coronary flow reserve, index of microcirculatory resistance, and fractional flow reserve, followed by vasoreactivity testing with acetylcholine. The primary endpoint was the mean difference in angina severity at 6 months. The authors concluded that stratified medical therapy, including an IDP with linked medical therapy, was routinely feasible and improved angina in patients with no obstructive CAD [17]. This strategy leads to marked and sustained angina improvement and better quality of life at 1 year following invasive coronary angiography [61]. The findings from this trial underline the need for an extended diagnostic framework when evaluating patients with INOCA. The correct diagnosis is a prerequisite for proper medical therapy and lifestyle intervention to increase quality of life in this population.

\section{Conclusions}

Microvascular dysfunction is responsible for angina in a substantial number of patients admitted for coronary angiogram. Diagnostic options are very limited in most centers, although these patients may have significant effects from cardiovascular risk reduction programs and tailored medical treatment, both in terms of symptoms and prognosis. Interventional cardiologists must lead the expansion of testing for microvascular angina so that the patients and the referring clinician have the correct diagnosis, which will aid in improved quality of life in these subjects.

\section{Conflicts of Interest}

William Fearon receives institutional research support from Abbott, Boston, and Medtronic and have consulting relationships with CathWorks and HeartFlow. Nico Pijls receives institutional research grants from Abbott and Exacath. Nico Pijls has received consultancy fees from Abbott and Opsens. Nico Pijls has minor equity in Philips, ASML, Heartflow, and General Electric. Todd Anderson and Alf Inge Larsen declare no conflicts of interest.

Alf I. Larsen

William F. Fearon

Todd J. Anderson

Nico Pijls 


\section{References}

[1] N. H. J. Pijls, P. van Schaardenburgh, G. Manoharan et al., "Percutaneous coronary intervention of functionally nonsignificant stenosis," Journal of the American College of Cardiology, vol. 49, no. 21, pp. 2105-2111, 2007.

[2] P. A. L. Tonino, B. De Bruyne, N. H. J. Pijls et al., "Fractional flow reserve versus angiography for guiding percutaneous coronary intervention," New England Journal of Medicine, vol. 360, no. 3, pp. 213-224, 2009.

[3] B. De Bruyne, W. F. Fearon, N. H. J. Pijls et al., "Fractional flow reserve-guided PCI for stable coronary artery disease," New England Journal of Medicine, vol. 371, no. 13, pp. 1208-1217, 2014.

[4] S. Sen, J. Escaned, I. S. Malik et al., "Development and validation of a new adenosine-independent index of stenosis severity from coronary wave-intensity analysis," Journal of the American College of Cardiology, vol. 59, no. 15, pp. 1392-1402, 2012.

[5] J. E. Davies, S. Sen, H. M. Dehbi et al., "Use of the instantaneous wave-free ratio or fractional flow reserve in PCI," The New England Journal of Medicine, vol. 376, pp. 1824-1834, 2017.

[6] M. Götberg, E. H. Christiansen, I. J. Gudmundsdottir, L. Sandhall, M. Danielewicz, and L. Jakobsen, "iFR-SWEDEHEART investigators. Instantaneous wave-free ratio versus fractional flow reserve to guide PCI," The New England Journal of Medicine, vol. 37, no. 19, pp. 1813-1823, 2017.

[7] M. R. Patel, J. H. Calhoon, G. J. Dehmer et al., "PKACC/AATS/ AHA/ASE/ASNC/SCAI/SCCT/STS 2017 appropriate use criteria for coronary revascularization in patients with stable ischemic heart disease: a report of the American College of Cardiology appropriate use criteria task force, American Association for Thoracic Surgery, American Heart Association, American Society of Echocardiography, American Society of Nuclear Cardiology, Society for Cardiovascular Angiography and Interventions, Society of Cardiovascular Computed Tomography, and Society of Thoracic Surgeons, J Am Coll Cardiol, 2017, 69, 2212-2241," Published Erratum Journal of the American College of Cardiology, vol. 71, no. 19, pp. 2279-2280, 2018.

[8] S. Ebihara, H. Otsuki, H. Arashi, J. Yamaguchi, and N. Hagiwara, "Rate pressure products affect the relationship between the fractional flow reserve and instantaneous wavefree ratio," Journal of Interventional Cardiology, vol. 21, Article ID 6230153, 2020.

[9] M. R. Patel, E. D. Peterson, D. Dai et al., "Low diagnostic yield of elective coronary angiography," New England Journal of Medicine, vol. 362, no. 10, pp. 886-895, 2010.

[10] N. B. Merz, B. D. Johnson, P. S. F. Kelsey et al., "Diagnostic, prognostic, and cost assessment of coronary artery disease in women," The American Journal of Managed Care, vol. 7, pp. 959-965, 2001.

[11] G. Niccoli, R. A. Montone, G. A. Lanza, and F. Crea, "Angina after percutaneous coronary intervention: the need for precision medicine," International Journal of Cardiology, vol. 248, pp. 14-19, 2017.

[12] T. J. Ford, E. Yii, N. Sidik et al., "Ischemia and no obstructive coronary artery disease: prevalence and correlates of coronary vasomotion disorders," Circulation. Cardiovascular Interventions, vol. 12, Article ID e008126, 2019.

[13] R. O. Cannon 3rd and S. E. Epstein, "“Microvascular angina” as a cause of chest pain with angiographically normal coronary arteries," The American Journal of Cardiology, vol. 61, no. 15, pp. 1338-1343, 1988.
[14] S. E. Epstein and R. O. Cannon, "Site of increased resistance to coronary flow in patients with angina pectoris and normal epicardial coronary arteries," Journal of the American College of Cardiology, vol. 8, no. 2, pp. 459-461, 1986.

[15] M. Prinzmetal, M. Kennamer, R. Merliss et al., "Angina pectoris I. A variant form of angina pectoris," The American Journal of Medicine, vol. 27, no. 3, pp. 375-388, 1959.

[16] D. S. Baim, "Coronary angiography," in Grossman's Cardiac Catheterization, Angiography, and Intervention, D. S. Baim, Ed., p. 215, 7th edition, Lippincott Williams \& Wilkins, Philadelphia, PA, USA, 2006.

[17] T. J. Ford, B. Stanley, R. Good et al., "Stratified medical therapy using invasive coronary function testing in angina," Journal of the American College of Cardiology, vol. 72, no. 23, pp. 2841-2855, 2018.

[18] A. I. Larsen, P. D. Galbraith, W. A. Ghali, C. M. Norris, M. M. Graham, and M. L. Knudtson, "Characteristics and outcomes of patients with acute myocardial infarction and angiographically normal coronary arteries," The American Journal of Cardiology, vol. 95, no. 2, pp. 261-263, 2005.

[19] S. H. Rezkalla and R. A. Kloner, "Coronary no-reflow phenomenon: from the experimental laboratory to the cardiac catheterization laboratory," Catheterization and Cardiovascular Interventions, vol. 72, no. 7, pp. 950-957, 2008.

[20] J. Shaw and T. Anderson, "Coronary endothelial dysfunction in non-obstructive coronary artery disease: risk, pathogenesis, diagnosis and therapy," Vascular Medicine, vol. 21, no. 2, pp. 146-155, 2016.

[21] K. Noma, Y. Kihara, and Y. Higashi, "Striking crosstalk of ROCK signaling with endothelial function," Journal of Cardiology, vol. 60, no. 1, pp. 1-6, 2012.

[22] M. Long, Z. Huang, X. Zhuang et al., "Association of inflammation and endothelial dysfunction with coronary microvascular resistance in patients with cardiac syndrome X." Arquivos brasileiros de cardiologia, vol. 109, pp. 397-403, 2017.

[23] P. Moreau, "Structure and reactivity of small arteries in aging," Cardiovascular Research, vol. 37, no. 1, pp. 247-253, 1998.

[24] I. Antony, A. Nitenberg, J.-M. Foult, and E. Aptecar, "Coronary vasodilator reserve in untreated and treated hypertensove patients with and without left ventricular hypertrophy," Journal of the American College of Cardiology, vol. 22, no. 2, pp. 514-520, 1993.

[25] P. J. Nahser, R. E. Brown, H. Oskarsson, M. D. Winniford, and J. D. Rossen, "Maximal coronary flow reserve and metabolic coronary vasodilation in patients with diabetes mellitus," Circulation, vol. 91, no. 3, pp. 635-640, 1995.

[26] N. Dagres, B. Saller, M. Haude et al., "Insulin sensitivity and coronary vasoreactivity: insulin sensitivity relates to adenosine-stimulated coronary flow response in human subjects," Clinical Endocrinology, vol. 61, no. 6, pp. 724-731, 2004.

[27] A. I. Larsen, R. Basran, T. Anderson, and D. Goodhart, "Large and small vessel vasoconstriction following coronary artery stenting," International Journal of Cardiology, vol. 113, no. 1, pp. 61-65, 2006.

[28] P. G. Camici and F. Crea, "Coronary microvascular dysfunction," New England Journal of Medicine, vol. 356, no. 8, pp. 830-840, 2007.

[29] J. M. Zelis, P. A. L. Tonino, N. H. J. Pijls et al., "Coronary microcirculation in aortic stenosis: pathophysiology, invasive assessment, and future directions," Journal of Interventional Cardiology, vol. 22, Article ID 4603169, 2020. 
[30] D. Baller, H. J. Bretschneider, and G. Hellige, "Validity of myocardial oxygen consumption parameters," Clinical Cardiology, vol. 2, no. 5, pp. 317-327, 1979.

[31] J. I. Hoffman and G. D. Buckberg, "The myocardial oxygen supply:demand index revisited," Journal of the American Heart Association, vol. 3, p. e000285, 2014.

[32] K. L. Gould and N. P. Johnson, "Quantitative coronary physiology for clinical management: the imaging standard," Current Cardiology Reports, vol. 18, no. 1, p. 9, 2016.

[33] D. C. J. Keulards, M. El Farissi, P. A. L. Tonino et al., "Thermodilution-based invasive assessment of absolute coronary blood flow and microvascular resistance: quantification of microvascular (Dys) function?" Journal of Interventional Cardiology, vol. 2020, Article ID 5024971, 7 pages, 2020.

[34] P. Xaplanteris, S. Fournier, D. C. J. Keulards et al., "Catheterbased measurements of absolute coronary blood flow and microvascular resistance: feasibility, safety, and reproducibility in humans," Circulation. Cardiovascular Interventions, vol. 11, Article ID e006194, 2018.

[35] B.-K. Lee, H.-S. Lim, W. F. Fearon et al., "Invasive evaluation of patients with angina in the absence of obstructive coronary artery disease," Circulation, vol. 131, no. 12, pp. 1054-1060, 2015.

[36] V. S. Pargaonkar, Y. Kobayashi, T. Kimura et al., "Accuracy of non-invasive stress testing in women and men with angina in the absence of obstructive coronary artery disease," International Journal of Cardiology, vol. 282, pp. 7-15, 2019.

[37] P. Meimoun and C. Tribouilloy, "Non-invasive assessment of coronary flow and coronary flow reserve by transthoracic Doppler echocardiography: a magic tool for the real world," European Journal of Echocardiography, vol. 9, no. 4, pp. 449-457, 2008.

[38] D. F. Bechsgaard and E. Prescott, "Transthoracic assessment of coronary flow velocity reserve; Practical approach to diagnostic testing in patients with angina and no obstructive coronary artery disease," Interventional Journal of Cardiology, vol. 2021, Article ID 6689312, 8 pages, 2021.

[39] P. Brainin, D. Frestad, and E. Prescott, "The prognostic value of coronary endothelial and microvascular dysfunction in subjects with normal or non-obstructive coronary artery disease: a systematic review and meta-analysis," International Journal of Cardiology, vol. 254, pp. 1-9, 2018.

[40] T. W. Hein, W. Wang, B. Zoghi, M. Muthuchamy, and L. Kuo, "Functional and molecular characterization of receptor subtypes mediating coronary microvascular dilation to adenosine," Journal of Molecular and Cellular Cardiology, vol. 33, no. 2, pp. 271-282, 2001.

[41] C. Caiati, C. Montaldo, N. Zedda et al., "Validation of a new noninvasive method (contrast-enhanced transthoracic second harmonic echo Doppler) for the evaluation of coronary flow reserve," Journal of the American College of Cardiology, vol. 34, no. 4, pp. 1193-1200, 1999.

[42] G. A. Lanza, A. Buffon, A. Sestito et al., "Relation between stress-induced myocardial perfusion defects on cardiovascular magnetic resonance and coronary microvascular dysfunction in patients with cardiac syndrome X," Journal of the American College of Cardiology, vol. 51, no. 4, pp. 466-472, 2008.

[43] F. Crea and G. A. Lanza, "Angina pectoris and normal coronary arteries: cardiac syndrome X," Heart, vol. 90, no. 4, pp. 457-463, 2004.

[44] R. Tyni-Lenne, S. Stryjan, B. Eriksson, M. Berglund, and C. Sylven, "Beneficial therapeutic effects of physical training and relaxation therapy in women with coronary syndrome X." Physiotherapy Research International, vol. 7, no. 1, pp. 35-43, 2002.
[45] D. S. Marks, S. Gudapati, L. M. Prisant et al., "Mortality in patients with microvascular disease," The Journal of Clinical Hypertension, vol. 6, no. 6, pp. 304-309, 2004.

[46] L. Jespersen, A. Hvelplund, S. Z. Abildstrom et al., "Stable angina pectoris with no obstructive coronary artery disease is associated with increased risks of major adverse cardiovascular events," European Heart Journal, vol. 33, no. 6, pp. 734-744, 2012.

[47] R. Sicari, F. Rigo, L. Cortigiani, S. Gherardi, M. Galderisi, and E. Picano, "Additive prognostic value of coronary flow reserve in patients with chest pain syndrome and normal or nearnormal coronary arteries," The American Journal of Cardiology, vol. 103, no. 5, pp. 626-631, 2009.

[48] A. K. Tahari, A. Lee, M. Rajaram et al., "Absolute myocardial flow quantification with 82Rb PET/CT: comparison of different software packages and methods," European Journal of Nuclear Medicine and Molecular Imaging, vol. 41, no. 1, pp. 126-135, 2014.

[49] V. L. Murthy, B. C. Lee, A. Sitek et al., "Comparison and prognostic validation of multiple methods of quantification of myocardial blood flow with 82Rb PET," Journal of Nuclear Medicine, vol. 55, no. 12, pp. 1952-1958, 2014.

[50] T. J. Anderson, F. Charbonneau, L. M. Title et al., "Microvascular function predicts cardiovascular events in primary prevention," Circulation, vol. 123, no. 2, pp. 163-169, 2011.

[51] B. H. Amundsen, U. Wisløff, and S. A. Slørdahl, "Exercise training in cardiovascular diseases," The Journal of the Norwegian Medical Association, vol. 127, pp. 446-448, 2007.

[52] F. M. Wise and J. M. Patrick, "Resistance exercise in cardiac rehabilitation," Clinical Rehabilitation, vol. 25, no. 12, pp. 1059-1065, 2011.

[53] J. Kaski and L. F. Valenzuela Garcia, "Therapeutic options for the management of patients with cardiac syndrome X," European Heart Journal, vol. 22, no. 4, pp. 283-293, 2001.

[54] T. K. Lim, A. J. Choy, F. Khan, J. J. Belch, A. D. Struthers, and C. C. Lang, "Therapeutic development in cardiac syndrome X: a need to target the underlying pathophysiology," Cardiovascular Therapeutics, vol. 27, no. 1, pp. 49-58, 2009.

[55] B. E. Eriksson, R. Tyni-Lennè, J. Svedenhag et al., "Physical training in syndrome X," Journal of the American College of Cardiology, vol. 36, no. 5, pp. 1619-1625, 2000.

[56] A. I. Larsen, T. Valborgland, J. Vegsundvag et al., "Aerobic high-intensity exercise training improves coronary flow reserve velocity and endothelial function in individuals with chest pain and normal coronary angiogram," European Heart Journal, vol. 36, no. 1154, 2015.

[57] I. O. Røysland, F. Friberg, B. Støre Brinchmann, S. Nordeide Svello, T. Valborgland, and A. I. Larsen, "Confronting one's vulnerability - patients with chest pain participating in a highintensity exercise programme," Journal of Clinical Nursing, vol. 26, pp. 2006-2015, 2017.

[58] D. Neglia, R. De Maria, S. Masi et al., "Effects of long-term treatment with carvedilol on myocardial blood flow in idiopathic dilated cardiomyopathy," Heart, vol. 93, no. 7, pp. 808-813, 2007.

[59] D. Neglia, E. Fommei, A. Varela-Carver et al., "Perindopril and indapamide reverse coronary microvascular remodelling and improve flow in arterial hypertension," Journal of $\mathrm{Hy}$ pertension, vol. 29, no. 2, pp. 364-372, 2011.

[60] "Ischemia-Intensive Medical Treatment Reduces Events in Women with Non-Obstructive CAD," 2021, https://clinicaltrials. gov/ct2/show/NCT03417388.

[61] T. J. Ford, B. Stanley, N. Sidik et al., "1-Year outcomes of angina management guided by invasive coronary function testing (CorMicA)," JACC: Cardiovascular Interventions, vol. 13, no. 1, pp. 33-45, 2020. 